\title{
Recommendations for neurological, obstetrical and gynaecological care in women with multiple sclerosis: a statement by a working group convened by the Section of Multiple Sclerosis and Neuroimmunology of the Polish Neurological Society
}

\author{
Alicja Kalinowska ${ }^{1}$, Alina Kułakowska ${ }^{2}$, Monika Adamczyk-Sowa ${ }^{3}, K^{2} z y s z t o f$ Czajkowski ${ }^{4}$, \\ Katarzyna Kurowska ${ }^{5}$, Bronisława Pietrzak ${ }^{6}$, Piotr Radziszewski ${ }^{7}, K$ Konrad Rejdak ${ }^{8}$, Halina Bartosik-Psujek ${ }^{9}$ \\ ${ }^{1}$ Department of Neurology, Division of Neurochemistry and Neuropathology, Poznan University of Medical Sciences, Poznan, Poland \\ ${ }^{2}$ Department of Neurology, Medical University of Bialystok, Bialystok, Poland \\ ${ }^{3}$ Department of Neurology, Zabrze Medical University of Silesia, Katowice, Poland \\ ${ }^{4}$ II Department of Obstetrics and Gynaecology, Medical University of Warsaw, Warsaw, Poland \\ ${ }^{5}$ Institute of Psychiatry and Neurology, Medical University of Warsaw, Warsaw, Poland \\ ${ }^{6}$ I Department of Obstetrics and Gynaecology, Medical University of Warsaw, Warsaw, Poland \\ ${ }^{7}$ Department of General, Oncological and Functional Urology, Medical University of Warsaw, Warsaw, Poland \\ ${ }^{8}$ Department of Neurology, Medical University of Lublin, Lublin, Poland \\ ${ }^{9}$ Medical Faculty, University of Rzeszow, Rzeszow, Poland
}

\begin{abstract}
Introduction. Multiple sclerosis (MS) is the most common non-traumatic neurological cause of disability in young adults, affecting women 1-3 times more often than men. Several specific challenges arise from the fact that young women diagnosed with MS often have to make decisions related to treatment and family planning at the same time. These issues are connected with fertility, the impact of pregnancy on disease course, the choice of pregnancy timing, and the optimal mode of disease-modifying therapy in the context of a planned pregnancy, contraception, urological complaints, and sexual dysfunction.

State of the art. While MS does not in itself adversely affect fertility, pregnancy or childbirth, pregnancy needs to be carefully planned. This requires the interdisciplinary co-operation of a neurologist, gynaecologist and psychologist. Data on the impact of disease-modifying drugs on foetal development are very limited, and none of these drugs is $100 \%$ safe during pregnancy. In the second and third trimesters, MS relapse rate decreases. Unfortunately, it increases within the first 3-6 months after delivery. Adequate disease control should be achieved before pregnancy, as relapse rate in the period of two years preceding pregnancy is one of the strongest predictive factors for post-partum relapses.

Clinical implications. The following is a statement by a working group of experts in neurology, gynaecology, obstetrics and urology, convened by the Section of Multiple Sclerosis and Neuroimmunology of the Polish Neurological Society, addressing the issues that are specific to the female MS population. The aim of this statement is to provide guidance in pregnancy planning and disease management, both during pregnancy and post-partum.

Future directions. This statement reflects expert opinion and is not intended to be read as guidelines. It rather provides up-to-date information on how to optimise care of female MS patients of childbearing age.
\end{abstract}

Key words: multiple sclerosis, women, pregnancy, childbearing age

(Neurol Neurochir Pol 2020; 54 (2): 125-137)

Address for correspondence: Alicja Kalinowska, Department of Neurology, Division of Neurochemistry and Neuropathology, Poznan University of Medical Sciences, Poznan, Poland, e-mail: akalinowskalyszczarz@ump.edu.pl 


\section{Introduction}

Multiple sclerosis (MS) is the most common neurological disease that causes disability in young adults [1]. The mean age of onset is about 30 years. Most studies report a higher prevalence of MS among women. The female-to-male ratio varies from 1.1 to 3 in relapsing-remitting MS (RRMS). Women and men are equally affected by primary progressive MS, which accounts for up to $10 \%$ of all MS cases [2]. In practice, MS is most prevalent among women of childbearing age. Studies show that only $21 \%$ of women decide to have offspring after the establishment of MS diagnosis, meaning that $79 \%$ of women with MS exclude motherhood $-34.5 \%$ justify their decision with disease-related factors (e.g. the fear that their condition will not allow them to raise children, the fear of passing the disease onto the child) [3]. In fact, the probability of a child whose parent is affected by MS developing the disease is low (approx. 2-3\%). Nonetheless, it is 15 times higher than in the general population [4]. Moreover, the disease does not adversely affect pregnancy or childbirth $[5,6]$. When one parent is affected by MS, the risk of the disease for each child is $2.5 \%$ [7]. When MS is diagnosed in both parents, the risk of MS in a child reaches $12.2 \%$ [8]. However, the disease is not hereditary.

\section{Epidemiology of multiple sclerosis}

The worldwide incidence and prevalence of MS are highly variable, both in terms of genetic and geographical factors. The number of patients with MS is estimated at 2.5 million worldwide, while in Europe it is around 630,000 patients [9]. After considering epidemiological data from neighbouring countries and research from selected regions of Poland, the estimated number of MS patients in Poland is approximately $45,000[10]$.

Nearly $5 \%$ of the world's population develops autoimmune diseases, $78 \%$ of whom are female [11]. The main theory explaining MS pathogenesis is based on the autoimmune concept [12]. Hence, it is not surprising that women are more affected by MS. The potential risk factors include genetic factors (targeted inactivation of the second $\mathrm{X}$ chromosome, foetal microchimerism) and hormonal factors (e.g. by oestrogen receptors).

Several environmental factors have a significant influence on disease distribution and prevalence [13], including Epstein-Barr virus (EBV) infection, exposure to sunlight and the level of Vitamin D, the effect of which may be particularly important in the prenatal period. A recent large prospective analysis of 77,330 women from the Danish National Birth Registry (1996-2002) [14] indicated new risk factors in the female population. An inverse relationship was observed between age at first menarche and the risk of MS. For each one-year increase in age at menarche, the risk of the disease was reduced by $13 \%$. However, the mechanism that could account for this relationship remains unknown.
Although genetic factors seem to be strongly associated with predisposition to the disease, specific genes related directly to MS risk have not been identified yet [15].

\section{Fertility of patients with multiple sclerosis}

There is no evidence for an association between menstrual cycle disorders and the development of MS. As in the general population, about $15 \%$ of couples with MS have problems with conception. The prevalence of sexual dysfunction in MS is estimated at 40\% among women and 65\% among men [16]. Studies show that the mean time from first attempts to conceive to the confirmation of pregnancy was seven months. However, this period was sometimes extended to nine months. This time was not statistically significantly different between the group of women with MS and the general population. According to Finnish data, the need for artificial insemination was greater in the group of women with MS [17]. Previous disease-modifying drugs (DMDs) had no impact on this period. Of note, unsuccessful attempts at in vitro fertilisation and administration of gonadotropin-releasing hormone agonists were associated with a higher risk of relapse. If the partner is affected by MS, ejaculation disorders and more frequent urinary tract infections (UTIs) are possible. Consequently, deterioration of semen parameters can be observed.

\section{Influence of pregnancy on the course of multiple sclerosis}

Pregnancy in Multiple Sclerosis (PRIMS), which included 254 women, was the first prospective study assessing the impact of pregnancy on MS [18]. It showed a decrease in relapses during pregnancy (by about 70\%) and an increase in the postpartum period (particularly the first three months). It was also found that the occurrence of relapses after pregnancy depended mainly on relapse activity before pregnancy.

Further studies $[19,20,21]$ did not show long-term effects of pregnancy on the natural course of the disease, and even suggested its protective effect by showing that women with a history of multiple births had lower disability and/or reached a particular level of disability during a longer period from the time of diagnosis. Such results could be related to the immunomodulatory effect of pregnancy [22].

A subsequent meta-analysis that combined the PRIMS data with 12 other studies [23] included 1,221 pregnancies. A reduction in the mean relapse rate was demonstrated, from 0.44 in the year before pregnancy to 0.26 during the entire pregnancy. The lowest rate $(0.18)$ was observed in the third trimester of pregnancy.

Hughes et al. further confirmed the results obtained by the PRIMS study [24]. They found no progression of disability among women in the analysed period, despite the fact that more relapses were observed immediately after delivery. 
The strongest predictive factor for post-partum MS relapse was the relapse rate in the two years preceding pregnancy, relapses during pregnancy, a disability score $\geq 2$ assessed by the Expanded Disability Status Scale (EDSS) at the time of conception, and a longer duration of the disease. Other studies have also indicated that women with a higher rate of relapses before pregnancy have a higher possibility of relapse during pregnancy [25-27]. Importantly, Hughes et al. showed that administration of DMDs in the two years before pregnancy reduced the risk of postpartum MS relapse by $45 \%$, and the authors suggested treatment should be continued until pregnancy is confirmed.

The protective effect on relapses during pregnancy is due to immune changes that occur in the female body which protect the developing foetus from the maternal immune system. The shift from a prevailing Th1 response to Th2-type response, with a simultaneous increase in the number of regulatory $\mathrm{T}$ cells, has been discussed [28]. However, this mechanism is probably more complex and also includes changes in cytotoxicity of CD56(bright) natural killer cells, CD4+ T cells producing interferon-gamma in the $\mathrm{CD} 4+/ \mathrm{CD} 8+$ ratio and in the concentration of interleukin 8.

\section{MS-modifying treatment}

Personalised therapy, which is considered the most appropriate approach, is based on selection of the proper drug in the right dose for the right patient to obtain the maximum effectiveness with the highest standards of safety. Previously, the choice of treatment for patients with MS was relatively simple, given that only single drugs were available. To date, 13 drugs with different mechanisms of action, efficacy and safety profile have been registered for MS. Personalisation of treatment in the context of family planning remains a crucial issue.

The general principles for selecting the first treatment for MS are based on the determination of the benefit/risk profile [29-32]. An escalation or an induction approach is chosen depending on the course (classic MS, or rapidly evolving severe, $\mathrm{RES}$, respectively). In the former, the therapy is started with the first-line DMD (parenteral medications i.e. beta-interferons and glatiramer acetate (GA), and oral medicines i.e. dimethyl fumarate or teriflunomide) and, if necessary, therapy is escalated when the treatment is not effective.

Patients with high disease activity may require more aggressive immunotherapies from the very beginning, with the use of natalizumab, fingolimod, cladribine or ocrelizumab (all are second-line drugs in Poland), the last two just recently approved for reimbursement in Poland, or alemtuzumab (recently restricted by the European Medicines Agency for use in adults with highly active RRMS despite adequate treatment with at least one disease-modifying therapy or if the disease is worsening rapidly with at least two disabling relapses per year and associated MRI activity).

\section{Pregnancy planning and DMDs in women with multiple sclerosis}

\section{Pregnancy planning}

Responsible motherhood requires pregnancy planning in MS patients [33-35]. Importantly, hormonal contraceptives do not adversely affect the course of the disease, and drugs containing high doses of oestrogens may have even beneficial anti-inflammatory effects $[36,37]$.

Pregnancy planning should be discussed with patients at diagnosis because (as shown by population studies in the USA) as many as $50 \%$ of pregnancies are unplanned, and the highest risk of abnormal foetal development occurs during weeks 4-8 of gestation [38]. Patients should be asked about whether they wish to have children and about the timing of pregnancy. The patient and her partner should have the chance to discuss objectively and impartially the issue of parenthood and the potential selection of the most appropriate method of contraception. The situation should be discussed not only with a neurologist but also with the members of the interdisciplinary team that should consist of an obstetrician-gynaecologist and a psychologist/psychiatrist.

From a neurological point of view, disease course and activity should be considered when the issue of motherhood is discussed. Ideally, there should be a minimum of one year between the time of diagnosis and decision-making about a planned pregnancy. This period is necessary to determine the course and activity of the disease. Although pregnancy has an immunomodulatory effect on MS, studies have shown that it should not be recommended in cases of MS with high activity and an aggressive course [39, 40]. A good response to DMD is defined as no evidence of disease activity (NEDA), both clinically and radiologically, for about two years. Obtaining such a response is the right moment to decide about becoming pregnant [41]. This moment, typically two years following the diagnosis, seems the most appropriate at which to risk discontinuation of therapy in most patients [42]. Obviously, the decision must be made individually by each patient. The particular type of DMD should also be considered.

Comorbidities and the symptoms of MS such as cognitive and emotional disorders should also be taken into account when the potential risk associated with pregnancy is assessed in patients.

Table 1 sets out the general criteria for safe pregnancy in patients with MS.

When a DMD is selected, attention should be paid to disease activity, the potential effect of the drug on pregnancy and foetal development, and the risk of relapse when the therapy is discontinued. Vitamin D supplementation must be considered when pregnancy is planned in MS patients. American guidelines recommend a dose of 1,000-2,000 U/ day [43]. Finnish studies showed that a reduced level of 25-hydroxyvitamin D [25 (OH) D] $(<12.02 \mathrm{ng} / \mathrm{mL})$ in the mother in early pregnancy was associated with a nearly doubled risk 
Table 1. Criteria for safe pregnancy in multiple sclerosis patients

1. Remission period.
2. No DMD for a period specific to each drug.
3. Favourable prognosis of the disease.
4. Intake of folic acid in a prophylactic dose for at least three months
before a planned pregnancy, and vitamin D supplementation in
the case of deficiency.
5. Considering pregnancy during administration of some DMDs in
individual cases.

DMD - disease-modifying drug

of MS in a child compared to the offspring of women with a normal blood level of vitamin D [44]. Patients with MS who plan pregnancy should follow the recommendations for all future mothers, including smoking cessation, alcohol abstinence, termination of stimulant use, following a balanced diet rich in omega- 3 polyunsaturated fatty acids, and the intake of folic acid.

\section{DMD in relation to pregnancy and family planning}

According to the recommendations of the European Medicines Agency (EMA) related to the impact of medical products on reproduction and lactation, a prospective follow-up of at least 300 , and preferably 1,000 , pregnancies is necessary to assess the safety of any given product [45]. The use of effective contraception (also after drug registration) is one of the requirements for participation in most clinical trials. During therapy, contraception is recommended. Therefore, the data on the impact of DMDs (especially newly registered) on foetal development are very limited.

Most DMDs used in the treatment of RRMS are classified in the Food and Drug Administration (FDA) pregnancy category $\mathrm{C}$, which means that animal reproductive studies have shown teratogenic or fatal effects on the foetus. However, there have been no well-controlled studies in women or there have been no adequate studies either in animals or humans. Classic cytostatic drugs (e.g. mitoxantrone) are in FDA pregnancy category $\mathrm{D}$ whereas category $\mathrm{X}$ was recently removed for teriflunomide. Glatiramer acetate (GA) was in category B. However, pregnancy has recently been removed from the list of contraindications (in the Summary of Product Characteristics) for a dose of 20-40 mg of glatiramer acetate, but also for interferon beta-1B subcutaneously every other day, interferon beta-1A subcutaneously three times a week, intramuscular interferon beta-1A every week, and interferon beta-1A subcutaneously every two weeks. It must be underlined that the safety data on GA during pregnancy is based on observations of the original product.

It should be noted that pregnancy categories A, B, C, D and X, introduced in 1979, have now been replaced with more detailed characteristics in the specific sections including e.g. pregnancy, delivery and the postpartum period, pregnancy registry, breastfeeding, reproductive potential and contraception [46].

Previously, all the guidelines recommended discontinuation of treatment before a planned pregnancy, with a 'wash-out' period of differing durations depending on the drug used. Nevertheless, based on real-world data (RWD), experienced neurologists specialising in MS have continued treatment with GA, interferon beta (IFN beta) or natalizumab until confirmation of pregnancy in those patients with a high risk of disease reactivation after treatment discontinuation [47, 48].

According to the most recent ECTRIMS/EAN Guidelines (2018) [29], patients should be informed that no DMDs should be used during pregnancy except for GA or interferons beta according to the registration documents. Continuation of treatment with GA or IFN beta should be considered until confirmation of pregnancy in women with a high risk of disease reactivation who plan pregnancy. In exceptional (active) cases, continuation of this therapy in pregnancy should also be considered.

Women with persistently high disease activity should be recommended to postpone pregnancy. If, however, the patient decides to become pregnant, or in an unplanned pregnancy, treatment with natalizumab should be considered after a thorough discussion with the patient regarding the potential implications.

Cladribine or alemtuzumab (taking into account its recent restrictions) could be an alternative option for patients with high disease activity who plan pregnancy, provided there is a 6-month (or 4-month for alemtuzumab) interval between the last dose and the planned conception.

The guidelines of the American Academy of Neurology (AAN 2018) [49] include similar recommendations. In general, DMDs should be discontinued before a planned pregnancy unless the risk of disease reactivation after discontinuation outweighs the risk of their use in pregnancy. It is also recommended to monitor childbearing plans in MS patients and pregnancy planning with the use of contraceptives during DMD therapy. Pregnancy planning should also apply to women whose partners are affected by MS. The AAN guidelines indicate the effect of cytostatic drugs on male fertility (cyclophosphamide) and the potential teratogenic effect by its influence on sperm (teriflunomide) [50]. It is recommended to discuss reproductive plans with MS men before the administration of these drugs. EAN/ECTRIMS guidelines do not provide recommendations for contraception in men treated with teriflunomide. According to the Summary of Product Characteristics, contraception is not advised for female partners of men who are treated with teriflunomide. Additionally, discontinuation of teriflunomide is not recommended for men before fatherhood [51].

Discontinuation of treatment that induces chronic immunomodulation or immunosuppression often leads to disease activation, as in the case of highly active natalizumab and fingolimod. On the other hand, therapies that 
result in reconstitution of the immune system, which are given for a short period of time and lead to long-lasting changes in the functioning of the immune system ('reprogramming the immune system'), may lead to long-term remission. These include alemtuzumab [52] and cladribine [53]. Due to the mechanism of action and dosing, drugs that cause reconstitution of the immune system seem to be a beneficial alternative to MS patients who plan to have children.

Table 2 sets out a summary of recommendations related to disease-modifying treatment in women with MS who plan pregnancy.

Table 3 sets out the characteristics of DMDs in the context of pregnancy, based on the current Summaries of Product Characteristics and reports from the literature.

\section{Impact of MS on pregnancy}

Studies have revealed that the percentage of miscarriages in MS patients is approximately $20 \%$, similar to the general population. The available data confirms that MS does not increase the risk of congenital disorders in offspring. The percentage of miscarriages remains at a similar level before and after the occurrence of the first symptoms of MS, and also among women who used DMDs and those who did not use DMDs. Although weakness, drowsiness and fatigue typically accompany pregnancy, in MS they may require neurological assessment.

A higher risk of preterm delivery, inhibited intrauterine growth in children, or intrauterine death has not been observed in the course of pregnancy in women with MS [54]. Other studies have indicated a slightly lower mean birth weight and a higher occurrence of small-for-gestational-age (SGA) births [55].
In the case of impaired mobility and paresis of the lower limbs, MS patients have an increased risk of thrombosis during pregnancy and puerperium. For this reason, compression stockings are recommended. Sometimes anticoagulants must be administered.

Single reports have indicated a higher incidence of UTIs during pregnancy in women with MS. Patients with functional disorders of the lower urinary tract secondary to MS must be aware of the exacerbation of these symptoms during pregnancy.

\section{Treatment of relapses in multiple sclerosis during pregnancy}

If an acute severe MS relapse occurs during pregnancy, treatment aimed at improving the general condition, accelerating recovery and creating the conditions for better development of pregnancy should be considered. The primary treatment of relapse is intravenous methylprednisolone $1 \mathrm{~g}$ daily for 3-5 days.

Non-fluorinated glucocorticosteroids (prednisone and methylprednisolone) poorly cross the placenta $(\leq 10 \%)$. They are mostly metabolised by placental 11-beta-hydroxysteroid dehydrogenase, which results in low concentrations in foetal blood and their use in the second and third trimesters is considered safe [56]. There are reports of possible lower birth weight and a slightly higher risk of preterm delivery. Methylprednisolone (iv) is in pregnancy category C. However, the risk of cleft lip and low birth weight is increased when it is used in the first trimester.

Fluorinated glucocorticoids (betamethasone, dexamethasone) are not recommended as they cross the placenta and are detected in the foetal blood at relatively high concentrations. These drugs may be used in short-term treatment in cases

Table 2. Summary of recommendations related to disease-modifying treatment in women with MS who plan pregnancy

1. In women with active disease, decision to start treatment with DMD should not be delayed, as long as patient is not pregnant yet. Effective contraception must be planned.

2. According to current ECTRIMS/EAN guidelines (2018), when a patient with MS plans pregnancy, patient's clinical condition must be established first (i.e. no disease activity for at least 1-2 years) and then attempts at pregnancy should be made.

3. Risk of relapse associated with drug withdrawal should be considered when deciding on DMD therapy (especially in the case of natalizumab and fingolimod). Possibility of effective elimination of the drug from serum (teriflunomide) should also be taken into consideration.

4. In the event of high risk of disease reactivation during pregnancy, continuation of treatment with IFN beta or GA may be considered until confirmation of pregnancy.

5. DMD therapy is not recommended during pregnancy. Continuation of therapy with GA (possible in Poland according to the Summary of Product Characteristics) or with interferons beta (possible according to the Summary of Product Characteristics for interferon beta 1-B subcutaneously every other day, interferon beta-1A subcutaneously three times weekly, intramuscular interferon beta-1A every week, interferon beta-1A subcutaneously every two weeks) may be considered in selected cases (in active disease) if the potential benefit outweighs risk associated with therapy.

6. Continuation of treatment with natalizumab during pregnancy should be considered (not possible under the Polish NHF drug programme due to no indications in Summary of Product Characteristics) after thorough discussion with patients on potential implications (e.g. haematological disorders of newborn) in the case of patients with high disease activity who are treated with natalizumab and intend to be pregnant when disease stabilisation is not achieved.

7. During an aggressive course of the disease, it is necessary to consider such therapy, the discontinuation of which will not be related to risk of reactivation (cladribine or alemtuzumab). During such treatment, patients can plan pregnancy four months after last dose of alemtuzumab and six months after last dose of cladribine.

DMD - disease-modifying drug; MS - multiple sclerosis; IFN — interferon; GA — glatiramer acetate; NHF — Polish National Health Fund 
Table 3. Characteristics of disease-modifying therapies in MS in the context of pregnancy based on the current summaries of the product characteristics and literature reports

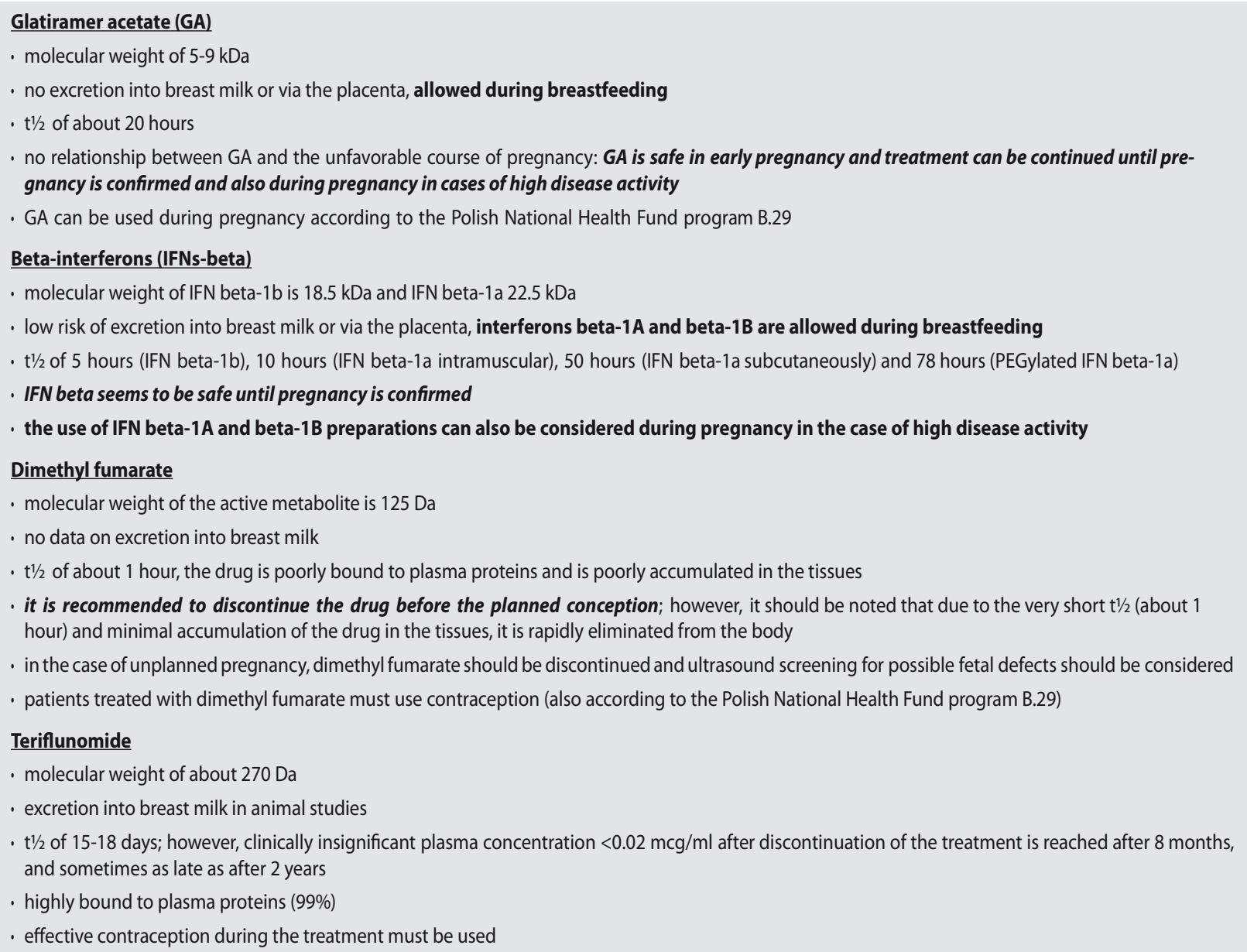

- if pregnancy is planned, treatment must be discontinued and the accelerated drug elimination procedure must be implemented, typically with cholestyramine ( $8 \mathrm{~g}, 3$ times/day for 11 days); drug concentration must be assessed in the blood using two determinations at an interval of at least 14 days; prior to the planned fertilization, patients have to wait for 1.5 months after the first confirmation of teriflunomide concentration $<0.02 \mathrm{mcg} / \mathrm{ml}$

- in the case of unintended pregnancy, the procedure of the accelerated elimination of the drug must be implemented immediately and ultrasound screening for fetal congenital defects must be performed;

- teriflunomide concentration in the semen of male patients is very low (100 times lower than blood concentration, after a dose of teriflunomide $14 \mathrm{mg}$ p.o.); low risk of transmission of teriflunomide by the male reproductive system, which could have a detrimental effect on the embryo or fetus

- patients treated with teriflunomide must use contraception (also according to the Polish National Health Fund program B.29)

\section{Natalizumab}

- molecular weight of $149 \mathrm{kDa}$

- $t \frac{1}{2}$ of $11 \pm 4$ days

- excreted into breast milk

- actively transported through the placental barrier (starting from the second trimester)

- currently, a slightly increased risk of miscarriage and fetal congenital defects cannot be ruled out - further follow-up is warranted

- there is a risk of hematological disorders in children of patients with high disease activity treated with natalizumab throughout the entire pregnancy

- there is a risk of disease activation, even with the rebound effect, after discontinuation of natalizumab and also during pregnancy in individual cases

- the conservative approach requires discontinuation of natalizumab 3 months before the planned pregnancy, which seems rather risky in view of disease activation

- a less conservative approach recommends discontinuation of natalizumab when attempting conception;

- the least conservative approach recommends discontinuation of the drug only when pregnancy is confirmed; when the disease becomes active during pregnancy, re-initiation of treatment with natalizumab is recommended at a dose of $300 \mathrm{mg}$ every 6 weeks and discontinuation of therapy before week 30 of pregnancy (in such cases, the newborn should be examined for hematological disorders)

- patients treated with natalizumab must use contraception (according to the Polish National Health Fund program B.46) 
Table 3. cont. Characteristics of disease-modifying therapies in MS in the context of pregnancy based on the current summaries of the product characteristics and literature reports

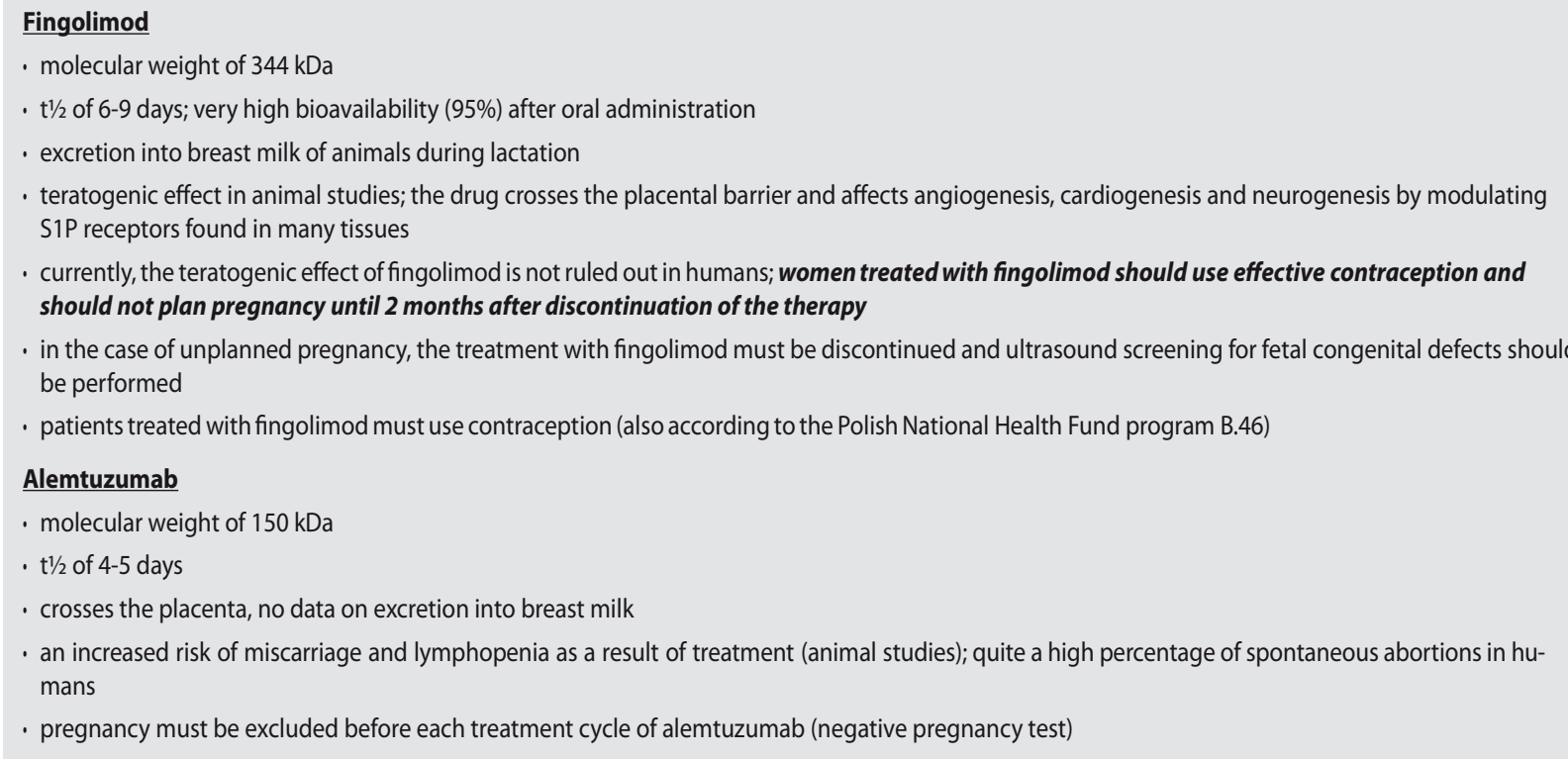

- contraception is recommended for 4 months following the last dose of alemtuzumab (the drug is completely eliminated from the body within 30 days after administration)

- patients must be monitored for secondary autoimmune complications and their potential impact on pregnancy in patients who become pregnant after the post-therapy contraception period

- patients treated with alemtuzumab must use contraception (also according to the Polish National Health Fund program B.29)

\section{Cladribine}

- molecular weight of $285.687 \mathrm{kDa}$

- no data on excretion into breast milk

- $\mathrm{t} 1 \frac{1}{2}$ of about 1 day; $20 \%$ bound to plasma proteins

- teratogenic effect in mice and rabbits; an adverse effect on gamete formation can be expected in humans

- pregnancy must be ruled out before drug administration

- cladribine-treated patients must use effective contraception during treatment and 6 months following the last administration of cladribine; mechanical contraception must be additionally used in patients who use systemic contraceptives during the treatment with cladribine and for 4 weeks immediately after the last dose of the drug

- women who become pregnant during treatment with cladribine should discontinue the treatment immediately and ultrasound screening for fetal congenital defects should be performed

- men treated with cladribine should use contraception during the treatment and for 6 months following the last dose to prevent pregnancy in their partners

\section{Ocrelizumab}

- molecular weight of $145 \mathrm{kDa}$

- no data on excretion into breast milk, the drug crosses the placental barrier

- $t \frac{1}{2}$ of 28 days (on average)

- no data on safety in pregnancy (FDA)

- decreased B-cells and transient lymphopenia in children of mothers treated with anti-CD20 antibodies during pregnancy

- women treated with ocrelizumab should use effective contraception during treatment and 12 months after the last dose

- the pregnancy registry is planned in patients treated with ocrelizumab

\section{Mitoxantrone}

- molecular weight of $517 \mathrm{kDa}$

- $t \frac{1}{2}$ of $23-215$ hours; slowly eliminated from the body - it is detectable in tissues after 9 months following the last administration; $78 \%$ bound to plasma proteins

- excretion into breast milk

- teratogenic effect in animals and humans

- women treated with mitoxantrone must use effective contraception; both men and women should not plan pregnancy within 6 months following the last administration

- the treatment with mitoxantrone can cause menstrual disorders, including permanent amenorrhea, particularly in women over 35 years of age ( $42 \%$ of cases); transient azoospermia (3-4 months) has been reported in men treated with mitoxantrone due to Hodgkin's lymphoma. 
of the onset of preterm delivery in order to accelerate foetal lung maturation.

If no improvement is observed after the first course of steroid therapy, another administration of methylprednisolone or intravenous immunoglobulins can be administered (category C; FDA). There is no established regimen for the use of immunoglobulins (IgGs). Typically, they are given in a dose of 0.2-0.4 g/kg body weight for five consecutive days. The use of IgGs in women with active disease during pregnancy to reduce the risk of relapse after delivery is currently not recommended. Such management, based on previous open studies which postulated a reduction in the increased number of relapses in the postpartum period by intravenous administration of $\operatorname{IgG}[57,58]$, was not confirmed in randomised studies [59, 60]. A prompt return to immunomodulatory treatment is recommended [61-63].

\section{Additional management in pregnancy}

\section{Treatment of infections}

Bladder dysfunction in women with MS may lead to a higher susceptibility to UTIs, which may result in exacerbation of the underlying disease. For this reason, in addition to routine urine tests, a urine culture should be performed in these patients at least once a month to detect asymptomatic UTIs. In both symptomatic and asymptomatic UTIs, the standard pharmacological treatment is recommended for 7-10 days, preferably according to the antibiogram. One week after the end of therapy, a follow-up urine culture should be performed. In the case of recurrent UTIs, pharmacological treatment should last for 14 days with subsequent prophylaxis of infections until the end of pregnancy by using one dose of an antibiotic or antimicrobial agent at night. Ultrasound investigation of the urinary tract is also recommended to detect other potential abnormalities.

\section{Maintenance of physical activity}

Physical activity and a balanced diet are the two most important factors improving the well-being and increasing the physical ability of MS patients. During pregnancy, physical activity is of importance not only to the well-being of pregnant women, but also since it allows reducing the risk of venous thromboembolic disease. Even though one of the predominant symptoms is increased fatigue, patients should not abandon active participation in everyday life. Most importantly, physical activity should be adapted to the stage of the disease and the overall physical capacity of the woman. Physical exercises must be safe and should improve their well-being. General physical activity and water exercises are recommended. In cases of reduced mobility, a prophylactic dose of low molecular weight heparin should be considered.

\section{Delivery, feeding and puerperium}

Multiple sclerosis is not a contraindication to natural delivery. However, impairment of physical fitness can make pushing more difficult. For this reason, surgical delivery is more prevalent in this group of women. Elective Caesarean section is performed in patients with severe motor disability.

Epidural anaesthesia during delivery is not contraindicated for patients with MS.

It does not affect the subsequent risk of relapse or progression of disability. It should be borne in mind that women with MS have a higher risk of perinatal depression [64].

Women with MS can breastfeed unless they are on a DMD. An influence of lactation on the relapse rate has not been observed $[65,66]$. In accordance with the current Summary of Product Characteristics, drug-related risk should always be considered when the therapy is continued during breastfeeding. Within four weeks after delivery, neurological assessment and optional magnetic resonance imaging are indicated to determine when DMD treatment is continued.

\section{Contraception in MS patients}

There is no doubt that highly effective contraception should be used during DMD treatment that is potentially teratogenic.

According to the guidelines of the World Health Organisation (WHO) $[67,68]$ and the recommendations of the American Centers for Disease Control and Prevention (CDC) [69], MS patients without immobilisation are included in Category 1 (no restriction for the use of the contraceptive method). Therefore, there are no contraindications for the use of intrauterine devices, levonorgestrel-releasing intrauterine systems, progestogen-only pills, agents containing oestrogens and progestogens such as contraceptive vaginal rings containing hormones, oral contraceptive pills, and transdermal contraceptive patches.

In this group of patients, only intramuscular administration of sustained-release medroxyprogesterone acetate (depot medroxyprogesterone acetate; DMPA) was included in Category 2, which means that the benefits of the method outweigh the potential risk. In the case of chronically immobilised patients, contraceptives containing silver, copper or levonorgestrel, progestogen-only pills and implants are included in Category 1 . The use of intramuscular sustained-release DMPA is classified in Category 2, and the combined contraceptive pill, contraceptive vaginal rings and patches in Category 3 , which means that their use is associated with a risk of complications. However, they are not absolutely contraindicated [70, 71].

It is known that the use of some contraceptive methods is associated with a risk of complications. The most common of these related to the combined contraceptive pill include venous thromboembolic disease, vascular diseases, deterioration in hypertension control, decreased libido, and decreased mood [67-69]. Long-term intramuscular injections of DMPA may result in decreased bone mass and the occurrence of abnormal genital bleeding. These adverse effects mostly disappear soon after drug discontinuation. However, subcutaneous 
etonogestrel implants may be safer in such situations as they do not cause bone loss.

\section{Urinary incontinence and urinary complaints in MS patients during pregnancy and after delivery}

Detrusor overactivity is the most common form of voiding disorder. It is usually associated with detrusor sphincter dyssynergia (functional obstruction). Underactivity or no contractile function of the detrusor is rare in the early form of the disease, whereas in advanced forms it is the most common cause of urinary retention and cessation. In a significant proportion of patients with MS, impaired lower urinary tract function changes with disease course. The intensity of the lower urinary tract dysfunction generally correlates with the level of disability.

Pregnancy and delivery increase lower urinary tract dysfunction, especially overactivity and impaired coordination between detrusor and sphincter. For this reason, increased urinary retention after voiding and the development of secondary UTIs are much more prevalent during pregnancy. The treatment of voiding disorders in MS patients depends on the type of voiding disorder and the degree of disability [72-74]. In addition, recurrent UTIs and the risk of renal failure should also be considered.

Lifestyle modifications (urinating by the clock, reasonable fluid intake, caffeine restriction) and bladder training (extending the time between voiding by 30 minutes at weekly intervals) are useful in the case of polyuria and urinary urgen$c y$ in the initial stage of the disease. The treatment includes antimuscarinics (tolterodine, solifenacin, oxybutynin) and beta-adrenergic agonists. However, in MS patients oxybutynin is not recommended as they are at risk of urinary retention. Mirabegron is the only representative of beta-adrenomimetics that does not significantly increase urinary retention due to the bladder-relaxant effect only in the filling phase. Botulinum toxin can also be used in the overactive bladder (100-300 units of onabotulinum toxin), with over $80 \%$ effectiveness. It can be obtained provided that patients can perform self-catheterisation after its use. A single administration of the toxin is usually sufficient for 6-9 months.

Functional bladder obstruction in MS patients results in increased urinary retention after voiding, weakened urinary stream and, consequently, detrusor muscle failure. Pharmacological treatment of functional bladder obstruction is poorly effective. Alpha-adrenolytic drugs (doxazosin, tamsulosin, silodosin, alfuzosin) are used to relax the bladder neck, whereas muscle relaxants (e.g. baclofen) are used to relax the external urethral sphincter. Pharmacological treatment of functional bladder obstruction is effective only in the initial period. Botulinum toxin administered to the external urethral sphincter is used in the treatment of functional obstruction $(\leq 100 \mathrm{U}$ of onabotulinum toxin, soft label use).
Self-catheterisation is the management of choice in the case of severe bladder obstruction with significant retention. The volume of residual urine which is associated with a higher prevalence of UTIs is above $150 \mathrm{ml}$. Volumes $>25 \%$ of the functional bladder capacity are considered clinically significant. Intermittent self-catheterisation is contraindicated when urinary retention volume is $<100 \mathrm{ml}$. The frequency of intermittent self-catheterisation is determined by diurnal diuresis, the functional bladder capacity, and residual urine after voiding. It is assumed that the volume of urine in the bladder should not exceed $400-500 \mathrm{ml}$. No-touch intermittent catheterisation is a recommended technique which involves single-use hydrophilic catheters. Small diameter catheters (10-12 Fr) of different lengths for women, men and children should be used. Catheters for women are shorter and more rigid than catheters for men [75].

Stress urinary incontinence associated with insufficient strength of the pelvic floor muscles is common in women with MS. It is the mechanical weakening of the suspensory apparatus of the urethra rather than damage to the innervation. The usual treatment of stress urinary incontinence is surgery and suburethral tape implantation. However, in patients with MS, complications should be expected in the form of increased overactivity, decreased bladder contractility, and urinary retention after voiding. Therefore, from the urological perspective, pregnancy in patients with MS should be carefully planned, preferably after intensive remission-targeted treatment [76].

\section{Conclusions and recommendations}

1. Pregnancy in MS patients should be planned, which requires interdisciplinary co-operation with a neurologist, a gynaecologist and sometimes also a psychologist.

2. Counselling for young women with MS is necessary with regards to effective methods of contraception. These are selected individually for the patient (oral, implant, or intrauterine contraceptive device).

3. No DMD is $100 \%$ safe during pregnancy. According to the Summary of the Product Characteristics, glatiramer acetate at a dose of $20 \mathrm{mg}$ subcutaneously every day or $40 \mathrm{mg}$ subcutaneously three times a week, interferon beta$-1 \mathrm{~B}$ subcutaneously every other day, interferon beta-1A subcutaneously three times a week, intramuscular interferon beta-1A every week, and interferon beta-1A subcutaneously every two weeks, are the only drugs that can be used during pregnancy (after considering the potential benefits and risks). The safety guidelines on GA during pregnancy are based on observations of the original GA.

4. In women with childbearing potential, it is necessary:

I. to determine the form of MS and to choose the treatment method accordingly and in line with the current guidelines.

II. to determine decisions related to pregnancy planning when: 
a) the patient wishes to become pregnant; pregnancy is recommended after the follow-up of disease activity and response to treatment for about two years after treatment onset. The drug elimination period must be considered when planning a pregnancy. In the case of high disease activity, neurological stabilisation should be achieved before planning pregnancy. Treatment that will not result in an increased risk of disease activation when therapy is discontinued should be considered (e.g. cladribine or alemtuzumab);

b) the patient does not want to become pregnant within the next two years or already has offspring, and uses effective contraception. In such cases, the reproductive age does not have to influence the choice of DMD;

c) the patient does not want to become pregnant within the next two years or already has offspring; neither does the patient comply with contraception guidelines. In such cases, the preferred therapy is GA or beta interferons.

5. Discontinuation of treatment in relation to a planned pregnancy is associated with a risk of increased disease activity, which is particularly related to patients treated with natalizumab and fingolimod.

6. Gynaecologists should be informed about possible adverse effects of DMD therapy by the attending neurologist.

7. In the second and third trimesters of pregnancy, relapses should be treated with nonfluorinated steroids (prednisone and methylprednisolone) in standard doses (0.5-1.0 g/day of methylprednisolone $i v$ for 3-5 days). In the first trimester, administration of steroids is associated with a risk of foetal defects and low birth weight. In such cases, intravenous human IgGs may be considered in a standard dose of $0.4 \mathrm{~g} / \mathrm{kg}$ body weight for five consecutive days.

8. Patients with MS have a higher risk of perinatal depression. Both the gynaecologist and the patient/family must be informed about the risk of depression and suicidal thoughts.

9. Within four weeks after delivery, a neurological assessment is indicated with magnetic resonance imaging (if needed) to determine when DMD treatment is re-administered. A rapid return to DMD treatment is recommended after pregnancy.

10. Pregnancy planning should also be related to women whose partner has been diagnosed with MS.

11. It is recommended to discuss reproductive plans with men diagnosed with MS before the inclusion of DMD treatment, and to consider sperm banking before treatment.

Ethical permission: Ethical approval was not necessary for preparation of this article

Funding: No direct funding was obtained to prepare this publication

Conflicts of interest: Alicja Kalinowska received grant funding from Novartis and received compensation for speaking and consulting services from Biogen, Bayer, Novartis, Roche, Merck, Teva, CSL Behring, Shire, and Sanofi-Genzyme; Alina Kułakowska received compensation for speaking and consulting services from Biogen, Bayer, Novartis, Roche, Merck, Teva, and Sanofi-Genzyme; Monika Adamczyk-Sowa received compensation for speaking and consulting services from Biogen, Bayer, Novartis, Roche, Merck, Teva and Sanofi-Genzyme; Bronislawa Pietrzak, Krzysztof Czajkowski and Piotr Radziszewski have no conflict of interest to declare; Katarzyna Kurowska received compensation for speaking and consulting services from Biogen, Bayer, Novartis, Roche, Merck, Teva, and Sanofi-Genzyme; Konrad Rejdak received compensation for speaking and consulting services from Biogen, Bayer, Novartis, Roche, Merck, Teva, and Sanofi-Genzyme; Halina Bartosik-Psujek received compensation for speaking and consulting services from Biogen, Bayer, Novartis, Roche, Merck, Teva, CSL Behring, and Sanofi-Genzyme.

\section{References}

1. Thompson AJ, Baranzini SE, Geurts J, et al. Multiple sclerosis. Lancet. 2018; 391(10130): 1622-1636, doi: 10.1016/S01406736(18)30481-1, indexed in Pubmed: 29576504.

2. D'hooghe MB, D'Hooghe T, De Keyser J. Female gender and reproductive factors affecting risk, relapses and progression in multiple sclerosis. Gynecol Obstet Invest. 2013; 75(2): 73-84, doi: 10.1159/000346319, indexed in Pubmed: 23343711.

3. Alwan S, Yee IM, Dybalski M, et al. Reproductive decision making after the diagnosis of multiple sclerosis (MS). Mult Scler. 2013; 19(3): 351-358, doi: 10.1177/1352458512452920, indexed in Pubmed: 22760102.

4. Compston A, Coles A. Multiple sclerosis. The Lancet. 2008; 372(9648): 1502-1517, doi: 10.1016/s0140-6736(08)61620-7.

5. Montalban X, Gold R, Thompson AJ, et al. Pregnancy, sex and hormonal factors in multiple sclerosis. Mult Scler. 2014; 20(5): 527-536, doi: 10.1177/1352458513519840, indexed in Pubmed: 24446387.

6. Kelly VM, Nelson LM, Chakravarty EF. Obstetric outcomes in women with multiple sclerosis and epilepsy. Neurology. 2009; 73(22): 1831 1836, doi: 10.1212/WNL.0b013e3181c3f27d, indexed in Pubmed: 19923552.

7. Dyment D, Ebers G, Sadovnick AD. Genetics of multiple sclerosis. The Lancet Neurology. 2004; 3(2): 104-110, doi: 10.1016/s1474$-4422(03) 00663-x$.

8. Ebers GC, Yee IM, Sadovnick AD, et al. Conjugal multiple sclerosis: population-based prevalence and recurrence risks in offspring. Canadian Collaborative Study Group. Ann Neurol. 2000; 48(6): 927-931, indexed in Pubmed: 11117550.

9. Kingwell $\mathrm{E}$, Marriott JJ, Jetté $\mathrm{N}$, et al. Incidence and prevalence of multiple sclerosis in Europe: a systematic review. BMC Neurol. 2013; 13: 128, doi: 10.1186/1471-2377-13-128, indexed in Pubmed: 24070256.

10. Kapica-Topczewska K, Brola W, Fudala M, et al. Prevalence of multiple sclerosis in Poland. Multiple Sclerosis and Related Disorders. 2018; 21: 51-55, doi: 10.1016/j.msard.2018.02.016.

11. Fairweather D, Frisancho-Kiss S, Rose NR. Sex differences in autoimmune disease from a pathological perspective. Am J Pathol. 2008; 173(3): 600-609, doi: 10.2353/ajpath.2008.071008, indexed in Pubmed: 18688037. 
12. Rejdak K, Jackson S, Giovannoni G. Multiple sclerosis: a practical overview for clinicians. Br Med Bull. 2010; 95: 79-104, doi: 10.1093/ bmb/ldq017, indexed in Pubmed: 20603280.

13. Ascherio A. Environmental factors in multiple sclerosis. Expert Rev Neurother. 2013; $13(12$ Suppl): 3-9, doi: 10.1586/14737175.2013.865866, indexed in Pubmed: 24289836.

14. Nielsen NM, Harpsøe M, Simonsen J, et al. Age at Menarche and Risk of Multiple Sclerosis: A Prospective Cohort Study Based on the Danish National Birth Cohort. Am J Epidemiol. 2017; 185(8): 712-719, doi: 10.1093/aje/kww160, indexed in Pubmed: 28369233.

15. Sawcer S, Compston A. The genetic analysis of multiple sclerosis in Europeans: concepts and design. J Neuroimmunol. 2003; 143(1-2): 13-16, doi: 10.1016/j.jneuroim.2003.08.035, indexed in Pubmed: 14575908.

16. Zorzon M, Zivadinov R, Bosco A, et al. Sexual dysfunction in multiple sclerosis: a case-control study. I. Frequency and comparison of groups. Mult Scler. 1999; 5(6): 418-427, doi: 10.1177/135245859900500i609, indexed in Pubmed: 10618699.

17. Jalkanen A, Alanen A, Airas L, et al. Finnish Multiple Sclerosis and Pregnancy Study Group. Pregnancy outcome in women with multiple sclerosis: results from a prospective nationwide study in Finland. Mult Scler. 2010; 16(8): 950-955, doi: 10.1177/1352458510372629, indexed in Pubmed: 20542921.

18. Confavreux C, Hutchinson M, Hours MM, et al. Rate of pregnancy-related relapse in multiple sclerosis. Pregnancy in Multiple Sclerosis Group. N Engl J Med. 1998; 339(5): 285-291, doi: 10.1056/NEJM199807303390501, indexed in Pubmed: 9682040.

19. D'hooghe MB, Haentjens P, Nagels G, et al. Menarche, oral contraceptives, pregnancy and progression of disability in relapsing onset and progressive onset multiple sclerosis. J Neurol. 2012; 259(5): 855-861, doi: 10.1007/s00415-011-6267-7, indexed in Pubmed: 21993617.

20. Masera S, Cavalla P, Prosperini L, et al. Parity is associated with a longer time to reach irreversible disability milestones in women with multiple sclerosis. Mult Scler. 2015; 21(10): 1291-1297, doi: 10.1177/1352458514561907, indexed in Pubmed: 25533293.

21. Runmarker B, Andersen 0 . Pregnancy is associated with a lower risk of onset and a better prognosis in multiple sclerosis. Brain. 1995; 118 ( Pt 1): 253-261, doi: 10.1093/brain/118.1.253, indexed in Pubmed: 7895009.

22. Gold SM, Voskuhl RR. Pregnancy and multiple sclerosis: from molecular mechanisms to clinical application. Semin Immunopathol. 2016; 38(6): 709-718, doi: 10.1007/s00281-016-0584-y, indexed in Pubmed: 27501960.

23. Finkelsztejn A, Brooks JBB, Paschoal FM, et al. What can we really tell women with multiple sclerosis regarding pregnancy? A systematic review and meta-analysis of the literature. BJOG. 2011; 118(7): 790-797, doi: 10.1111/j.1471-0528.2011.02931.x, indexed in Pubmed: 21401856.

24. Hughes SE, Spelman T, Gray OM, et al. MSBase study group. Predictors and dynamics of postpartum relapses in women with multiple sclerosis. Mult Scler. 2014; 20(6): 739-746, doi: 10.1177/1352458513507816, indexed in Pubmed: 24107309.

25. Keyhanian K, Davoudi V, Etemadifar M, et al. Better prognosis of multiple sclerosis in patients who experienced a full-term pregnancy. Eur Neurol. 2012; 68(3): 150-155, doi: 10.1159/000338847, indexed in Pubmed: 22854684.

26. Portaccio E, Ghezzi A, Hakiki B, et al. MS Study Group of the Italian Neurological Society. Postpartum relapses increase the risk of dis- ability progression in multiple sclerosis: the role of disease modifying drugs. J Neurol Neurosurg Psychiatry. 2014; 85(8): 845-850, doi: 10.1136/jnnp-2013-306054, indexed in Pubmed: 24403285.

27. Vukusic S, Confavreux C. Pregnancy and multiple sclerosis: the children of PRIMS. Clin Neurol Neurosurg. 2006; 108(3): 266-270, doi: 10.1016/j.clineuro.2005.11.016, indexed in Pubmed: 16413965.

28. Airas L, Saraste M, Rinta S, et al. Finnish Multiple Sclerosis and Pregnancy Study Group. Immunoregulatory factors in multiple sclerosis patients during and after pregnancy: relevance of natural killer cells. Clin Exp Immunol. 2008; 151(2): 235-243, doi: 10.1111/j.1365-2249.2007.03555.x, indexed in Pubmed: 18062798.

29. Montalban X, Gold R, Thompson AJ, et al. ECTRIMS/EAN guideline on the pharmacological treatment of people with multiple sclerosis. Eur J Neurol. 2018; 25(2): 215-237, doi: 10.1111/ene.13536, indexed in Pubmed: 29352526.

30. Torkildsen $\emptyset$, Myhr KM, Bø L. Disease-modifying treatments for multiple sclerosis - a review of approved medications. European Journal of Neurology. 2015; 23: 18-27, doi: 10.1111/ene.12883.

31. Soelberg Sorensen P. Safety concerns and risk management of multiple sclerosis therapies. Acta Neurol Scand. 2017; 136(3): 168-186, doi: 10.1111/ane.12712, indexed in Pubmed: 27891572.

32. Comi G, Radaelli M, Soelberg Sørensen P. Evolving concepts in the treatment of relapsing multiple sclerosis. Lancet. 2017; 389(10076): 1347-1356, doi: 10.1016/S0140-6736(16)32388-1, indexed in Pubmed: 27889192.

33. Thöne J, Thiel S, Gold R, et al. Treatment of multiple sclerosis during pregnancy - safety considerations. Expert Opin Drug Saf. 2017; 16(5): 523-534, doi: 10.1080/14740338.2017.1311321, indexed in Pubmed: 28333552.

34. Amato MP, Bertolotto A, Brunelli R, et al. Management of pregnancyrelated issues in multiple sclerosis patients: the need for an interdisciplinary approach. Neurol Sci. 2017; 38(10): 1849-1858, doi: 10.1007/s10072-017-3081-8, indexed in Pubmed: 28770366.

35. Voskuhl R, Momtazee C. Pregnancy: Effect on Multiple Sclerosis, Treatment Considerations, and Breastfeeding. Neurotherapeutics. 2017; 14(4): 974-984, doi: 10.1007/s13311-017-0562-7, indexed in Pubmed: 28766273.

36. McCombe PA, Greer JM. Female reproductive issues in multiple sclerosis. Mult Scler. 2013; 19(4): 392-402, doi: 10.1177/1352458512452331, indexed in Pubmed: 22733837.

37. Pozzilli C, De Giglio L, Barletta VT, et al. Oral contraceptives combined with interferon $\beta$ in multiple sclerosis. Neurol Neuroimmunol Neuroinflamm. 2015; 2(4): e120, doi: 10.1212/NXI.0000000000000120, indexed in Pubmed: 26140279.

38. Finer LB, Zolna MR. Unintended pregnancy in the United States: incidence and disparities, 2006. Contraception. 2011; 84(5): 478485, doi: 10.1016/j.contraception.2011.07.013, indexed in Pubmed: 22018121.

39. Coyle PK. Multiple sclerosis and pregnancy prescriptions. Expert Opin Drug Saf. 2014; 13(12): 1565-1568, doi: 10.1517/14740338.2014.973848, indexed in Pubmed: 25406727.

40. Ferrero S, Pretta S, Ragni N. Multiple sclerosis: management issues during pregnancy. Eur J Obstet Gynecol Reprod Biol. 2004; 115(1): 3-9, doi: 10.1016/j.ejogrb.2003.10.020, indexed in Pubmed: 15223156.

41. Banwell B, Giovannoni G, Hawkes C, et al. Editors' welcome and a working definition for a multiple sclerosis cure. Mult Scler Relat Disord. 2013; 2(2): 65-67, doi: 10.1016/j.msard.2012.12.001, indexed in Pubmed: 25877624. 
42. Amato MP, Bertolotto A, Brunelli R, et al. Management of pregnancyrelated issues in multiple sclerosis patients: the need for an interdisciplinary approach. Neurol Sci. 2017; 38(10): 1849-1858, doi: 10.1007/s10072-017-3081-8, indexed in Pubmed: 28770366.

43. ACOG Committee on Obstetric Practice. ACOG Committee Opinion No. 495: Vitamin D: Screening and supplementation during pregnancy. Obstet Gynecol. 2011; 118(1): 197-198, doi: 10.1097/ AOG.0b013e318227f06b, indexed in Pubmed: 21691184.

44. Munger KL, Åivo J, Hongell K, et al. Vitamin D Status During Pregnancy and Risk of Multiple Sclerosis in Offspring of Women in the Finnish Maternity Cohort. JAMA Neurol. 2016; 73(5): 515-519, doi: 10.1001/ jamaneurol.2015.4800, indexed in Pubmed: 26953778.

45. European Medicines Agency (EMA) Evaluation of Medicines for Human Use. Guideline on risk assessment of medical products on human reproduction and lactation: from data to labeling. http://www. ema.europa.eu/docs/en_GB/document_library/Scientific_guideline/2009/09/WC500003307.pdf (Google Scholar, London 24 July 2008).

46. FDA/CDER SBIA Chronicles. Drugs in Pregnancy and Lactation: Improved Benefit-Risk Information. January 22, 2015 . http://www.fda. gov/downloads/Drugs/DevelopmentApprovalProcess/SmallBusinessAssistance/UCM431132.pd (Accessed July 25, 2016).

47. Thöne J, Thiel S, Gold R, et al. Treatment of multiple sclerosis during pregnancy - safety considerations. Expert Opin Drug Saf. 2017; 16(5): 523-534, doi: 10.1080/14740338.2017.1311321, indexed in Pubmed: 28333552.

48. Amato MP, Portaccio E. Fertility, pregnancy and childbirth in patients with multiple sclerosis: impact of disease-modifying drugs. CNS Drugs. 2015; 29(3): 207-220, doi: 10.1007/s40263-015-0238-y, indexed in Pubmed: 25773609.

49. Rae-Grant A, Day GS, Marrie RA, et al. Practice guideline recommendations summary: Disease-modifying therapies for adults with multiple sclerosis: Report of the Guideline Development, Dissemination, and Implementation Subcommittee of the American Academy of Neurology. Neurology. 2018; 90(17): 777-788, doi: 10.1212/WNL.0000000000005347, indexed in Pubmed: 29686116.

50. Thöne J, Thiel S, Gold R, et al. Treatment of multiple sclerosis during pregnancy - safety considerations. Expert Opin Drug Saf. 2017; 16(5): 523-534, doi: 10.1080/14740338.2017.1311321, indexed in Pubmed: 28333552.

51. https://ec.europa.eu/health/documents/community-register/201/20151216133467/anx_133467_pl.pdf.

52. Hill-Cawthorne GA, Button T, Tuohy 0 , et al. Long term lymphocyte reconstitution after alemtuzumab treatment of multiple sclerosis. J Neurol Neurosurg Psychiatry. 2012; 83(3): 298-304, doi: 10.1136/ jnnp-2011-300826, indexed in Pubmed: 22056965.

53. Giovannoni G. Cladribine to Treat Relapsing Forms of Multiple Sclerosis. Neurotherapeutics. 2017; 14(4): 874-887, doi: 10.1007/s13311017-0573-4, indexed in Pubmed: 29168160.

54. Finkelsztejn A, Brooks JBB, Paschoal FM, et al. What can we really tell women with multiple sclerosis regarding pregnancy? A systematic review and meta-analysis of the literature. BJOG. 2011; 118(7): 790 797, doi: 10.1111/j.1471-0528.2011.02931.x, indexed in Pubmed: 21401856.

55. Dahl J, Myhr KM, Daltveit AK, et al. Pregnancy, delivery, and birth outcome in women with multiple sclerosis. Neurology. 2005; 65(12): 1961-1963, doi: 10.1212/01.wnl.0000188898.02018.95, indexed in Pubmed: 16380620.
56. Losy J, Bartosik-Psujek H, Członkowska A, et al. Leczenie stwardnienia rozsianego. Zalecenia Polskiego Towarzystwa ogicznego Pol. Przegl Neurol. 2016; 12(2): 80-95.

57. Haas J. High dose IVIG in the post partum period for prevention of exacerbations in MS. Mult Scler. 2000; 6 Suppl 2: S18-20; discussion S33, indexed in Pubmed: 11188773.

58. Achiron A, Rotstein Z, Noy S, et al. Intravenous immunoglobulin treatment in the prevention of childbirth-associated acute exacerbations in multiple sclerosis: a pilot study. J Neurol. 1996; 243(1): 25-28, doi: 10.1007/bf00878527, indexed in Pubmed: 8869383.

59. Coyle PK, Christie S, Fodor P, et al. Women Neurologists MS Initiative. Multiple sclerosis gender issues: clinical practices of women neurologists. Mult Scler. 2004; 10(5): 582-588, doi: 10.1191/1352458504ms1083oa, indexed in Pubmed: 15471377.

60. Hellwig K, Beste C, Schimrigk S, et al. Immunomodulation and postpartum relapses in patients with multiple sclerosis. Ther Adv Neurol Disord. 2009; 2(1): 7-11, doi: 10.1177/1756285608100416, indexed in Pubmed: 21180639.

61. Alroughani R, Altintas A, Al Jumah M, et al. Pregnancy and the Use of Disease-Modifying Therapies in Patients with Multiple Sclerosis: Benefits versus Risks. Mult Scler Int. 2016; 2016: 1034912, doi: 10.1155/2016/1034912, indexed in Pubmed: 28078140.

62. Vaughn C, Bushra A, Kolb C, et al. An Update on the Use of DiseaseModifying Therapy in Pregnant Patients with Multiple Sclerosis. CNS Drugs. 2018; 32(2): 161-178, doi: 10.1007/s40263-018-0496-6, indexed in Pubmed: 29508244.

63. Alroughani R, Alowayesh MS, Ahmed SF, et al. Relapse occurrence in women with multiple sclerosis during pregnancy in the new treatment era. Neurology. 2018; 90(10): e840-e846, doi: 10.1212/ WNL.0000000000005065, indexed in Pubmed: 29429970.

64. Razaz N, Tremlett H, Marrie RA, et al. Peripartum depression in parents with multiple sclerosis and psychiatric disorders in children. Mult Scler. 2016; 22(14): 1830-1840, doi: 10.1177/1352458516631037, indexed in Pubmed: 26903008.

65. Airas L, Jalkanen A, Alanen A, et al. Breast-feeding, postpartum and prepregnancy disease activity in multiple sclerosis. Neurology. 2010; 75(5): 474-476, doi: 10.1212/WNL.0b013e3181eb5860, indexed in Pubmed: 20679640.

66. Portaccio E, Ghezzi A, Hakiki B, et al. Breastfeeding is not related to postpartum relapses in multiple sclerosis. Neurology. 2011; 77(2): 145-150, doi: 10.1212/wnl.0b013e318224afc9.

67. World Health Organization. Medical Eligibility Criteria for Contraceptive Use. 4th ed. Geneva: World Health Organization; 2009. http://whqlibdoc.who.int/publications/2009/ 9789241563888eng.pdf (Accessed June 8, 2010).

68. Curtis KM, Jamieson DJ, Peterson HB, et al. Adaptation of the World Health Organization's medical eligibility criteria for contraceptive use for use in the United States. Contraception. 2010; 82(1): 3-9, doi: 10.1016/j.contraception.2010.02.014, indexed in Pubmed: 20682138

69. Centers for Disease Control and Prevention. US Medical Eligibility Criteria for Contraceptive Use, 2010. Adapted from the World Health Organization Medical Eligibility Criteria for Contraceptive Use, 4th edition. MMWR Early Release. May 28. 2010; 59: 186.

70. Houtchens MK, Zapata LB, Curtis KM, et al. Contraception for women with multiple sclerosis: Guidance for healthcare providers. Mult Scler. 2017; 23(6): 757-764, doi: 10.1177/1352458517701314, indexed in Pubmed: 28338393.

71. Kempe P, Hammar M, Brynhildsen J. Symptoms of multiple sclerosis during use of combined hormonal contraception. Eur J Obstet Gynecol 
Reprod Biol. 2015; 193: 1-4, doi: 10.1016/j.ejogrb.2015.06.030, indexed in Pubmed: 26196655.

72. Hinson JL, Boone TB. Urodynamics and multiple sclerosis. Urol Clin North Am. 1996; 23(3): 475-481, doi: 10.1016/s00940143(05)70326-8, indexed in Pubmed: 8701560.

73. Ukkonen M, Elovaara I, Dastidar $\mathrm{P}$, et al. Urodynamic findings in primary progressive multiple sclerosis are associated with increased volumes of plaques and atrophy in the central nervous system. Acta Neurol Scand. 2004; 109(2): 100-105, doi: 10.1034/j.1600-0404.2003.00184.x, indexed in Pubmed: 14705971.
74. Ciancio S, Mutchnik S, Rivera V, et al. Urodynamic pattern changes in multiple sclerosis. Urology. 2001; 57(2): 239-245, doi: 10.1016/ s0090-4295(00)01070-0.

75. Emmanuel A, Gajewski J, Hamid R, et al. Sievert K-D, Wyndaele JJ. Neurologic Urinary and Faecal Incontinence. In: Abrams P, Cardozo L, Wagg A, Wein A (Eds). Incontinence 6th Edition. ICl-ICS. International Continence Society, Bristol. : 2017.

76. Chapple C. Bladder dysfunction, gynaecological aspects of urology. Current Opinion in Urology. 1996; 6(4): 169-170, doi: 10.1097/00042307-199607000-00001. 